\title{
Quest for the extra degree of freedom in $f(T)$ gravity
}

\author{
Rafael Ferraro ${ }^{1,2, *}$ and María José Guzmán ${ }^{3, \dagger}$ \\ ${ }^{1}$ Instituto de Astronomía y Física del Espacio (IAFE, CONICET-UBA), Casilla de Correo 67, \\ Sucursal 28, 1428 Buenos Aires, Argentina \\ ${ }^{2}$ Departamento de Física, Facultad de Ciencias Exactas y Naturales, Universidad de Buenos Aires, \\ Ciudad Universitaria, Pabellón I, 1428 Buenos Aires, Argentina \\ ${ }^{3}$ Instituto de Física de La Plata (IFLP, CONICET-UNLP), C. C. 67, 1900 La Plata, Argentina
}

(Received 6 November 2018; published 27 December 2018)

\begin{abstract}
It has recently been shown that $f(T)$ gravity has $\frac{n(n-3)}{2}+1$ physical degrees of freedom (d.o.f.) in $n$ dimensions, contrary to previous claims. The simplest physical interpretation of this fact is that the theory possesses a scalar d.o.f. This is the case of $f(R)$ gravity, a theory that can be understood in the Einstein frame as general relativity plus a scalaron. The scalar field that represents the extra d.o.f. in $f(T)$ gravity encodes information about the parallelization of the spacetime, which is detected through a reinterpretation of the equations of motion in both the teleparallel Jordan and Einstein frames. The trace of the equations of motion in $f(T)$ gravity shows the propagation of the scalar d.o.f., giving an accurate proof of its existence. We also provide a simple toy model of a physical system with rotational pseudoinvariance, like $f(T)$ gravity, which gives insights into the physical interpretation of the extra d.o.f. We discuss some implications and unusual features of the previously worked out Hamiltonian formalism for $f(T)$ gravity. Finally we show some mathematical tools to implement the Hamiltonian formulation in the Einstein frame of $f(T)$ gravity, which exhibits some problems that should be addressed in future works.
\end{abstract}

DOI: $10.1103 /$ PhysRevD.98.124037

\section{INTRODUCTION}

Gravitational theories based on a spacetime with absolute parallelism are extensions of general relativity (GR) that are being thoroughly studied in the literature. They intend to solve the main problems of cosmology like the hypothesis of dark matter, the accelerated expansion of the Universe and the inflation paradigm, as well as theoretical problems of general relativity like the emergence of singularities, and the quest for a quantum field theory of gravity. Although teleparallel and modified teleparallel theories of gravity have a long history, their applications in the realm of cosmology started to being considered just recently in the context of teleparallelism à la Born-Infeld [1]. The original motivation of this work was to obtain an early accelerated expansion of the Universe without resorting to an inflaton field. This scheme addresses the problems of general relativity at the high-energy regime, which has been studied and discussed to some extent in subsequent work [2-8]. The article [1] is a particular case of the socalled $f(T)$ gravity, a slight variation of teleparallel models that has also been proposed as an alternative explanation of the late-time accelerated expansion of the Universe [9]. Since then, there has been a growing interest in this kind of

\footnotetext{
ferraro@iafe.uba.ar

†mjguzman@fisica.unlp.edu.ar
}

theories [10-44] and several other gravity models based in parallelized spacetimes have been developed [45-50]. Modified teleparallel theories of gravity on all its forms are expected to be an effective theory of a more fundamental physical theory valid at energy regimes near the Planck energy.

There is one issue about $f(T)$ gravity that has given rise to disputes in the literature: the fact that it is not invariant under local Lorentz transformations of the tetrad field. This fact is usually interpreted as the selection of preferred frames that parallelize the spacetime, which implies that the theory contains extra degrees of freedom (d.o.f.) if compared with GR. Although this issue could be tackled by replacing the standard Weitzenböck connection with a general one to obtain a covariant version of the theory [51-53], the number of d.o.f. would remain unchanged since the same dynamical equations should be expected in such covariant versions of $f(T)$ gravity.

A recent work has established that $f(T)$ gravity contains one extra d.o.f. compared with the teleparallel equivalent of general relativity (TEGR) in arbitrary dimension [42]. Although there could be many physical objects possessing a single d.o.f., the easiest interpretation would be that the theory is equivalent to the teleparallel equivalent of general relativity plus a scalar field minimally coupled to it. However, a very known fact is that $f(T)$ gravity does not possess the analog of an Einstein frame, at least not in 
the way that it occurs in $f(R)$ gravity $[15,35]$. Therefore, it is timely to discuss the interpretation of the physical d.o.f. of $f(T)$ gravity in the light of its $f(R)$ counterpart. We will analyze whether the scalar field interpretation is valid both in the teleparallel Jordan and Einstein equivalent frames and look for further insights in their respective Hamiltonian formulations.

This work is organized as follows. In Sec. II we will review the basics of $f(R)$ gravity and the interpretation of the additional d.o.f. on this theory, together with the definition of the Jordan and Einstein frames. In Sec. III we will introduce the TEGR and its simplest generalization, $f(T)$ gravity. In Sec. IV we will analyze the issue of the extra d.o.f. in $f(T)$ gravity through the comparison of the teleparallel Jordan and the Einstein frames actions and their equations of motion. In Sec. V we will present a mechanical system with rotational pseudoinvariance, which is useful to exemplify several uncommon features of $f(T)$ gravity and will help to understand the physical meaning of the extra d.o.f. In Sec. VI we will discuss some features of the Hamiltonian formalism for $f(T)$ gravity developed in [42]. In Sec. VII we will introduce the Hamiltonian formalism for the teleparallel Einstein frame and discuss the doability of the Dirac-Bergmann algorithm in two different approaches. Finally we will dedicate Sec. VIII to the conclusions.

\section{II. $f(R)$ GRAVITY}

One of the most studied and simplest modifications of general relativity consists in replacing the GR Lagrangian $R$ with a function $f(R)$ of the scalar curvature. Such a gravitational theory is described by the action

$$
S=-\frac{1}{2 \kappa} \int d^{4} x \sqrt{-g} f(R)+S_{\text {matter }},
$$

where $\kappa=8 \pi G$. In (1) and throughout this work we adopt the convention $(+,-,-,-)$ for the metric signature. The variation of the action (1) gives rise to the following fourthorder dynamical equations:

$f^{\prime}(R) R_{\mu \nu}-\frac{1}{2} f(R) g_{\mu \nu}-\left[\nabla_{\mu} \nabla_{\nu}-g_{\mu \nu} \square\right] f^{\prime}(R)=\kappa \mathcal{T}_{\mu \nu}$,

where $f^{\prime}(R)=d f / d R$ and $\mathcal{T}_{\mu \nu}$ is the matter stress-energy tensor. We see that $f^{\prime}(R)$ acts as a renormalization of the gravitational constant $\kappa$, and therefore only functions satisfying $f^{\prime}(R)>0$ should be considered; besides it should be $f^{\prime \prime}(R)>0$ in order to avoid instabilities [54-57].

Taking the trace of the equations of motion (2) we can see that the Ricci scalar $R$ is dynamically determined by the trace $\mathcal{T}$ of the energy-momentum tensor $\mathcal{T}_{\mu \nu}$ through the second-order equation

$$
f^{\prime}(R) R-2 f(R)+3 \square f^{\prime}(R)=\kappa \mathcal{T}
$$

(in four dimensions). While Einstein's equations imply that $R$ vanishes in vacuum, this equation possesses nontrivial propagating solutions in vacuum, so suggesting the existence of a new d.o.f. related to $R$ [or $f^{\prime}(R)$ ]. The presence of additional d.o.f. in $f(R)$ gravity is foreseeable, since the Cauchy data for the higher (fourth) order of the differential equations involve more functions to be chosen on the Cauchy surface. The issue of how many new d.o.f. are involved in $f(R)$ gravity can be better understood in the socalled Jordan frame, which we proceed to review.

\section{A. Jordan frame}

Equations (2) are fourth-order differential equations for the metric. But, alternatively, one could regard the set (2), (3) as second-order equations for the metric and the scalar object $f^{\prime}(R)$. Without any harm let us change the notation to

$$
\phi \equiv f^{\prime}(R), \quad V(\phi) \equiv R \phi-f(R),
$$

to rewrite Eqs. (2) as [58]

$R_{\mu \nu}-\frac{1}{2} g_{\mu \nu} R=\frac{\kappa}{\phi} \mathcal{T}_{\mu \nu}-\frac{g_{\mu \nu}}{2 \phi} V(\phi)+\frac{1}{\phi}\left[\nabla_{\mu} \nabla_{\nu} \phi-g_{\mu \nu} \square \phi\right]$.

Equation (4) defines the Legendre transform of $f(R)$. Therefore one also obtains

$$
R=V^{\prime}(\phi) .
$$

Equations (5) and (6) can be recognized as the dynamical equations associated with the action

$S_{J F}\left[g_{\mu \nu}, \phi\right]=-\frac{1}{2 \kappa} \int d^{4} x \sqrt{-g}[\phi R-V(\phi)]+S_{\text {matter }}$

(which resembles a Brans-Dicke action with $\omega_{B D}=0$ and a nontrivial potential). By varying the action (7) with respect to $\phi$ one gets Eq. (6), which says the scalar $\phi$ is a function of the scalar curvature $R$. This result implies that the Lagrangian in (7) is dynamically equivalent to the Legendre transform of the function $V(\phi)$; then, it can be rewritten as a function of $R$ uniquely:

$$
f(R)=\phi R-V(\phi),
$$

which completes the full circle of the equivalence between the actions (7) and (1). In fact, Eqs. (4), (6) and (8) contain the entire mechanism of the Legendre transform and its inverse $\left(f^{\prime \prime} \neq 0\right.$ in order that the transformation be invertible). The action (7) is said to be the Jordan frame representation of $f(R)$ gravity.

Notice that one can use the result (6) to write the trace of Eq. (5) as a wave equation for a self-interacting scalar field $\phi$ : 


$$
3 \square \phi-\phi^{3}\left[\phi^{-2} V(\phi)\right]^{\prime}=\kappa \mathcal{T} .
$$

From this perspective, $\phi$ looks like a scalar field minimally coupled to the metric $g_{\mu \nu}$, but coupled to the matter. On the other hand, Eqs. (5) are Einstein equations sourced by the matter and the scalar field. Thus, $f(R)$ gravity can be rephrased as a scalar-tensor theory with second-order dynamical equations [59-61]. As dynamical equations for the metric $g_{\mu \nu}$, Eqs. (5) keep all the symmetries contained in Einstein equations ${ }^{1}$; so, they describe two d.o.f. in four dimensions. Besides, there is one extra d.o.f. described by the second-order equation (9).

\section{B. Einstein frame}

An even clearer understanding of the extra scalar d.o.f. in $f(R)$ gravity is achieved by working in the so-called Einstein frame. In this representation the extra d.o.f. is distilled in the action as a scalar field possessing its own kinetic term. This goal is attained by means of a conformal transformation of the metric together with a redefinition of the scalar field:

$$
g_{\mu \nu} \rightarrow \tilde{g}_{\mu \nu}=\phi g_{\mu \nu}, \quad \phi \rightarrow \tilde{\phi}=\sqrt{\frac{3}{2 \kappa}} \ln \phi
$$

(then it is $\sqrt{-g}=\phi^{-2} \sqrt{-\tilde{g}}$ ). Thus, applying the relation for the scalar curvatures of conformally related metrics

$$
\phi \tilde{R}=R-\frac{3}{2} g^{\mu \nu} \partial_{\mu} \ln \phi \partial_{\nu} \ln \phi-3 \square \ln \phi,
$$

the action $S_{J F}$ in (7) is rewritten as

$$
\begin{aligned}
S_{E F}\left[\tilde{g}_{\mu \nu}, \tilde{\phi}\right]= & \int d^{4} x \sqrt{-\tilde{g}}\left(-\frac{\tilde{R}}{2 \kappa}+\frac{1}{2} \tilde{g}^{\mu \nu} \partial_{\mu} \tilde{\phi} \partial_{\nu} \tilde{\phi}-U(\tilde{\phi})\right) \\
& +S_{\text {matter }},
\end{aligned}
$$

where the new potential $U(\tilde{\phi})$ is

$$
U(\tilde{\phi})=-\frac{V(\phi)}{2 \kappa \phi^{2}}=\frac{f(R)-R f^{\prime}(R)}{2 \kappa f^{\prime}(R)^{2}} .
$$

The action (12) describes a tensor field $\tilde{g}_{\mu \nu}$ governed by Einstein equations and a minimally coupled scalar field $\tilde{\phi}$ governed by a Klein-Gordon equation that includes a selfinteraction [62-65]. Therefore, the Einstein frame has the virtue of explicitly showing the extra d.o.f. at the level of the action, since $S_{E F}$ is the sum of the Einstein-Hilbert action and the scalar field action. Of course $\tilde{g}_{\mu \nu}$ and $\tilde{\phi}$ are not the original tensor and scalar fields; but it does not matter for the purpose of counting d.o.f. Anyway, once the

\footnotetext{
${ }^{1}$ In particular, the automatic conservation of $\mathcal{T}_{\mu \nu}$ is guaranteed.
}

Einstein frame dynamical equations are solved, one can always return to the original metric $g_{\mu \nu}$ by means of Eq. (10).

Our very aim is to investigate whether the issue of the extra d.o.f. in $f(T)$ gravity can be addressed in analogy with the $f(R)$ gravity case. For this, we will introduce $f(T)$ theories of gravity and its progenitor, the teleparallel equivalent of general relativity.

\section{TELEPARALLEL AND $\boldsymbol{f}(\boldsymbol{T})$ GRAVITY}

\section{A. Teleparallel gravity}

In order to build a dynamical theory for a field of orthonormal frames, we begin by defining a manifold $M$ and a basis of vectors $\left\{\mathbf{e}_{a}\right\}$ in the tangent space $T_{p}(M)$. We also define a dual basis $\left\{\mathbf{E}^{a}\right\}$ in the cotangent space $T_{p}^{*}(M)$ such that $\mathbf{E}^{a}\left(\mathbf{e}_{b}\right)=\delta_{b}^{a}$. If expanded in a coordinate basis,

$$
\mathbf{e}_{a}=e_{a}^{\mu} \partial_{\mu}, \quad \mathbf{E}^{a}=E_{\mu}^{a} d x^{\mu},
$$

the duality relationships are written as

$$
E_{\mu}^{a} e_{b}^{\mu}=\delta_{b}^{a}, \quad e_{a}^{\mu} E_{\nu}^{a}=\delta_{\nu}^{\mu} .
$$

We will use Greek letters $\mu, \nu, \ldots=0, \ldots, n-1$ for spacetime coordinate indices and Latin letters $a, b, \ldots=0, \ldots$, $n-1$ for tangent space or Lorentz indices. A vielbein is a basis that encodes the metric structure through the following relation:

$$
\mathbf{g}=\eta_{a b} \mathbf{E}^{a} \otimes \mathbf{E}^{b}
$$

then the inverse expression

$$
\mathbf{e}_{a} \cdot \mathbf{e}_{b}=\mathbf{g}\left(\mathbf{e}_{a}, \mathbf{e}_{b}\right)=\eta_{a b}
$$

states that the vielbein is an orthonormal basis. In $n=4$ we have a vierbein or tetrad. The expressions (16) and (17) can be alternatively written in coordinate language as

$$
g_{\mu \nu}=\eta_{a b} E_{\mu}^{a} E_{\nu}^{b}, \quad \eta_{a b}=g_{\mu \nu} e_{a}^{\mu} e_{b}^{\nu} .
$$

This notation also implies that $\sqrt{-g}=\operatorname{det}\left(E_{\mu}^{a}\right) \equiv E$, and also $e \equiv \operatorname{det}\left(e_{a}^{\mu}\right)=E^{-1}$. We can construct a dynamical theory for the spacetime through the tetrad field, since it encodes the metric tensor and henceforth the geometry of the spacetime. To get equations of motion equivalent to Einstein equations we use the TEGR action,

$$
S=\frac{1}{2 \kappa} \int d^{4} x E T+S_{\text {matter }}
$$

where $T$ is a local Lorentz pseudoinvariant made up of the torsion of the Weitzenböck connection 


$$
T_{\nu \rho}^{\mu}=e_{a}^{\mu}\left(\partial_{\nu} E_{\rho}^{a}-\partial_{\rho} E_{\nu}^{a}\right)
$$

In terms of this torsion, $T$ is written as

$$
T \equiv T^{\rho}{ }_{\mu \nu} S_{\rho}{ }^{\mu \nu},
$$

where the object $S_{\rho}{ }^{\mu \nu}$ is called the superpotential and is given by

$S_{\rho}^{\mu \nu} \equiv-\frac{1}{4}\left(T_{\rho \nu}^{\mu \nu}-T_{\rho}^{\nu \mu}-T_{\rho}{ }^{\mu \nu}\right)+\frac{1}{2} T_{\sigma}{ }^{\sigma \mu} \delta_{\rho}^{\nu}-\frac{1}{2} T_{\sigma}{ }^{\sigma \nu} \delta_{\rho}^{\mu}$.

GR and TEGR are equivalent theories because the GR Lagrangian $R$ differs from the TEGR Lagrangian in a boundary term,

$$
R=-T+2 e \partial_{\mu}\left(E T^{\mu}\right),
$$

where $R$ is the Ricci scalar of the Levi-Civita connection associated with the metric (18) and $T^{\mu} \equiv T_{\rho}^{\rho}{ }_{\rho}^{\mu}$ is the vector part of the torsion.

By varying the action (19) with respect to the tetrad field we obtain the following dynamical equations:

$4 e \partial_{\mu}\left(E e_{a}^{\lambda} S_{\lambda}{ }^{\mu \nu}\right)+4 e_{a}^{\lambda} T^{\rho}{ }_{\mu \lambda} S_{\rho}{ }^{\mu \nu}-e_{a}^{\nu} T=-2 \kappa e_{a}^{\lambda} \mathcal{T}_{\lambda}{ }^{\nu}$,

which prove to be equivalent to Einstein equations for the metric (18). In particular, the trace of (24) in four dimensions is

$$
2 e E_{\nu}^{a} \partial_{\mu}\left(E e_{a}^{\lambda} S_{\lambda}^{\mu \nu}\right)=-\kappa \mathcal{T},
$$

where $\mathcal{T}$ is the trace of the energy-momentum tensor. However it can be proven that

$$
2 e E_{\nu}^{a} \partial_{\mu}\left(E e_{a}^{\lambda} S_{\lambda}{ }^{\mu \nu}\right)=-T+2 e \partial_{\mu}\left(E T^{\mu}\right) .
$$

Therefore it results

$$
-T+2 e \partial_{\mu}\left(E T^{\mu}\right)=-\kappa \mathcal{T} .
$$

The lhs is $R$, as we know from the Lagrangian equivalence (23). Thus we retrieve the trace of Einstein equations.

\section{B. $f(T)$ gravity}

The teleparallel equivalent of general relativity can be utilized as an underlying framework for a more general class of modified gravities. The simplest choice is to generalize the TEGR Lagrangian through an arbitrary function of the torsion scalar $T$; this gives rise to the socalled $f(T)$ gravity. The action for this theory is given by

$$
S\left[E_{\mu}^{a}\right]=\frac{1}{2 \kappa} \int d^{4} x E f(T)+S_{\text {matter }},
$$

where $T$ is the Weitzenböck or torsion scalar, which is quadratic in first-order derivatives of the tetrad field. This fact alone implies that the equations of motion of this theory will always be second order, which is an advantage over other modified gravities [as metric $f(R)$ gravity]. In fact, by varying the action (28) with respect to the tetrad, it results [26]

$$
\begin{aligned}
& 4 e \partial_{\mu}\left(f^{\prime}(T) E e_{a}^{\lambda} S_{\lambda}{ }^{\mu \nu}\right)+4 f^{\prime}(T) e_{a}^{\lambda} T^{\sigma}{ }_{\mu \lambda} S_{\sigma}{ }^{\mu \nu}-e_{a}^{\nu} f(T) \\
& \quad=-2 \kappa e_{a}^{\lambda} \mathcal{T}_{\lambda}{ }^{\nu} .
\end{aligned}
$$

Equation (29) shows that $f^{\prime}(T)$ renormalizes the gravitational constant $\kappa$ and the volume $E$. It has been known since the beginning of the theory that the action of $f(T)$ gravity and their equations of motion are sensitive to local Lorentz transformations in the tangent space of the spacetime manifold: given a tetrad $\mathbf{E}^{a}$ that solves Eqs. (29), the Lorentz transformed tetrad $\mathbf{E}^{a^{\prime}}=\Lambda^{a^{\prime}}{ }_{a}(x) \mathbf{E}^{a}$ will not necessarily solve them. The departure from the local Lorentz invariance can be already recognized in the action; the torsion scalar $T$ is invariant under global Lorentz transformations of the tetrad, but local transformations will provide it with terms containing derivatives of the Lorentz matrices $\Lambda^{a^{\prime}}{ }_{a}(x)$. For TEGR this fact is harmless; according to Eq. (23), $T$ is equal to $-R$-which depends only on the (locally invariant) metric - plus a boundary term which is the responsible for the lack of local invariance. But a boundary term is irrelevant for the dynamics in the TEGR action (19). On the contrary, the most general functional form $f$ in (28) is not linear; thus the four-divergence term remains encapsulated in $f$, and the local Lorentz invariance is definitely lost.

\section{The premetric formulation of TEGR}

We will find useful in later sections and future work to rewrite several mathematical objects by getting rid of the metric tensor in favor of the tetrad field and the Minkowski metric. As it was firstly introduced in [66], the TEGR Lagrangian can be written in a compact form like

$$
L=E T=\frac{1}{2} E \partial_{\mu} E^{a}{ }_{\nu} \partial_{\rho} E^{b}{ }_{\lambda} e_{c}^{\mu} e_{e}^{\nu} e_{d}^{\rho} e_{f}^{\lambda} M_{a b}{ }^{c e d f},
$$

where $M_{a b}{ }^{c e d f}$ is the supermetric, the Lorentz invariant tensor given by

$M_{a b}^{c e d f}=2 \eta_{a b} \eta^{c[d} \eta^{f] e}-4 \delta_{a}^{[d} \eta^{f][c} \delta_{b}^{e]}+8 \delta_{a}^{[c} \eta^{e][d} \delta_{b}^{f]}$.

This object is nothing more than a constitutive tensor, that is the object that relates the excitation field and the field strength of any physical theory $[67,68]$. This result can be derived alternatively by writing both $T^{\rho}{ }_{\mu \nu}$ and $S_{\rho}{ }^{\mu \nu}$ in the premetric formalism. Observing that 
$T^{\rho}{ }_{\mu \nu}=e_{a}^{\rho}\left(\partial_{\mu} E_{\nu}^{a}-\partial_{\nu} E_{\mu}^{a}\right)=\partial_{\sigma} E_{\lambda}^{a} e_{a}^{\rho} e_{b}^{\sigma} e_{c}^{\lambda} E_{\mu}^{d} E_{\nu}^{e}\left(\delta_{d}^{b} \delta_{e}^{c}-\delta_{e}^{b} \delta_{d}^{c}\right)$,

we can rewrite the superpotential $S_{\rho}{ }^{\mu \nu}$ defined in (22) as

$$
S_{\rho}^{\mu \nu}=E_{\rho}^{d} \partial_{\sigma} E_{\lambda}^{a} e_{b}^{\sigma} e_{c}^{\lambda} e_{e}^{\mu} e_{f}^{\nu} S_{a d}{ }^{b e c f},
$$

where we have defined

$$
\begin{aligned}
S_{a d}^{b e c f}= & \eta_{a d} \eta^{e[b} \eta^{c] f}-\eta^{f[b} \delta_{d}^{c]} \delta_{a}^{e}+\eta^{e[b} \delta_{d}^{c]} \delta_{a}^{f}+2 \delta_{a}^{b} \eta^{c[e} \delta_{d}^{f]} \\
& -2 \delta_{a}^{c} \eta^{b[e} \delta_{d}^{f]} .
\end{aligned}
$$

Performing the corresponding algebra, it is not difficult to check that, up to a factor $E$, the multiplication of (32) and (34) gives (30). This is in virtue of the simple result

$$
S_{b a}{ }^{d c f e}-S_{b a}{ }^{d e f c}=M_{a b}{ }^{c e d f} .
$$

We will need for later to rewrite the four-divergence $\partial_{\mu}\left(E T^{\mu}\right)$, that distinguishes the (Levi-Civita) curvature scalar from the torsion scalar. This is written as

$$
\partial_{\mu}\left(E T^{\mu}\right)=\partial_{\mu}\left(E g^{\mu \nu} e_{a}^{\rho}\left(\partial_{\rho} E_{\nu}^{a}-\partial_{\nu} E_{\rho}^{a}\right)\right) .
$$

Taking into account that $\partial_{\mu} E=E e_{a}^{\lambda} \partial_{\mu} E_{\lambda}^{a}$, and replacing the metric tensor by its tetrad counterpart, in the end it is obtained

$$
\begin{aligned}
B \equiv e \partial_{\mu}\left(E T^{\mu}\right)= & \frac{1}{2} \partial_{\mu} E_{\nu}^{a} \partial_{\rho} E_{\lambda}^{b} e_{c}^{\mu} e_{e}^{\nu} e_{d}^{\rho} e_{f}^{\lambda} B_{a b} \text { cedf } \\
& +\partial_{\mu} \partial_{\rho} E_{\nu}^{a} e_{b}^{\mu} e_{c}^{\nu} e_{d}^{\rho}\left(\delta_{a}^{d} \eta^{b c}-\delta_{a}^{c} \eta^{b d}\right),
\end{aligned}
$$

where the analog of the constitutive tensor for the fourdivergence is written as

$B_{a b}{ }^{c e d f}=4 \eta^{c d} \delta_{a}^{[f} \delta_{b}^{e]}+4 \eta^{c e} \delta_{a}^{[d} \delta_{b}^{f]}+4 \eta^{c f} \delta_{a}^{[e} \delta_{b}^{d]}+4 \delta_{a}^{c} \eta^{e[d} \delta_{b}^{f]}$.

Remarkably this object does not have the same antisymmetry properties as $M_{a b}$ cedf .

\section{Approaches on the degrees of freedom of $\boldsymbol{f}(\boldsymbol{T})$ gravity}

While in $f(R)$ gravity the increase of the number of d.o.f. manifests itself through the increase of the order of the differential equations, in $f(T)$ gravity the dynamical equations remain as second-order equations. Instead, the $f(T)$ dynamical equations have less gauge freedom than the TEGR equations, because not all the local Lorentz transformations of the tetrad are symmetries of the dynamics. Less gauge freedom implies more genuine d.o.f.
There are several approaches to the question of the number and nature of the d.o.f. of $f(T)$ gravity, which do not necessarily coincide with their outcomes. The Hamiltonian analysis of $f(T)$ gravity presented in [23], based on Maluf's Hamiltonian formulation of TEGR [69], gives $n-1$ extra d.o.f. for $f(T)$ gravity in arbitrary dimension. In [32] it is performed a simple Hamiltonian analysis based on the number of pairs of Lorentz constraints that would become second class. The authors establish that $f(T)$ gravity might have five, four or two d.o.f. based on the possibility that six, four or two Lorentz constraints become second class. The enforcement of the vanishing of the Riemann tensor through the introduction of Lagrange multipliers has been analyzed with detail in [50]. This strategy attempts to avoid a particular choice of the spin connection but adds a large quantity of Lagrange multipliers that are nevertheless determined at the end of the procedure. Other approaches that need further development in order to give a characterization of the d.o.f. are the study of the remnant group of local Lorentz symmetries [34], the null tetrad approach $[33,38]$ and the discussion on the covariantization of the theory [51-53], among others. Finally, the recent work performed in [42] suggests that there exists only one additional d.o.f. in arbitrary dimension. The counting of d.o.f. obtained in this work is not the same as the one presented in [23], nor falls in any of the categories sketched in [32]. However, if the theory has one rightful extra d.o.f., it could be similar to the scalaron that appears in $f(R)$ theories of gravity. We will discuss the possibility of finding a scalar d.o.f. in the equations of motion of $f(T)$ gravity in the next section.

\section{THE EXTRA DEGREE OF FREEDOM OF $\boldsymbol{f}(\boldsymbol{T})$ GRAVITY}

\section{A. Jordan frame}

The previous discussions, and the comparison with $f(R)$ gravity in Sec. II, could suggest taking a look at the Jordan action as a way to understand the nature of the extra d.o.f. This approach is successful in $f(R)$ gravity because it decouples the equations at the same time that it reduces their differential order. However, in $f(T)$ gravity the equations of motion (29) are already second order. Besides, by tracing them we do not succeed in isolating the dynamics of some scalar d.o.f.; in fact, in four dimensions the trace is

$e E_{\nu}^{a} \partial_{\mu}\left(f^{\prime}(T) E e_{a}^{\lambda} S_{\lambda}^{\mu \nu}\right)+f^{\prime}(T) T-f(T)=-\frac{\kappa}{2} \mathcal{T}$,

that can be rewritten as

$$
\begin{aligned}
& e \partial_{\mu}\left(f^{\prime}(T) E E_{\nu}^{a} e_{a}^{\lambda} S_{\lambda}{ }^{\mu \nu}\right)-f^{\prime}(T) e_{a}^{\lambda} S_{\lambda}{ }^{\mu \nu} \partial_{\mu} E_{\nu}^{a}+f^{\prime}(T) T-f(T) \\
& =-\frac{\kappa}{2} \mathcal{T} .
\end{aligned}
$$

Let us now consider the dynamics in the Jordan frame, which is defined by the Legendre transform 


$$
\phi=f^{\prime}(T), \quad V(\phi)=T \phi-f(T),
$$

also implying that it is

$$
T=V^{\prime}(\phi)
$$

Thus the dynamical equations (29) $\mathrm{read}^{2}$

$$
\begin{aligned}
& 4 \phi^{-1} e \partial_{\mu}\left(\phi E e_{a}^{\lambda} S_{\lambda}{ }^{\mu \nu}\right)+4 e_{a}^{\lambda} T^{\sigma}{ }_{\mu \lambda} S_{\sigma}{ }^{\mu \nu}-e_{a}^{\nu} T \\
& =-\frac{2 \kappa}{\phi} e_{a}^{\lambda} \mathcal{T}_{\lambda}{ }^{\nu}+e_{a}^{\nu} \frac{V(\phi)}{\phi}
\end{aligned}
$$

which keep the structure of TEGR field equations, except for the renormalizations of the gravitational constant $\kappa$ and the volume $E$, and the term $\phi^{-1} V(\phi)$ playing the role of a local cosmological constant. Equations (43) and (42) can be obtained by varying the action

$$
S_{J F}\left[E_{\mu}^{a}, \phi\right]=\frac{1}{2 \kappa} \int d^{4} x E[\phi T-V(\phi)]+S_{\text {matter }} .
$$

In particular, Eq. (42) results from varying with respect to $\phi$, which means that the Lagrangian is dynamically equivalent to the Legendre transform of $V(\phi)$, and so it is a function $f(T)$ such that $\phi=f^{\prime}(T)$. Thus, the action (44) is dynamically equivalent to the action (28).

Let us now analyze the trace of the dynamical equations. In four dimensions it results that $S_{\nu}{ }^{\mu \nu}=T_{\nu}{ }^{\nu \mu}=T^{\mu}$. Besides, it is $e_{a}^{\lambda} S_{\lambda}^{\mu \nu} \partial_{\mu} E_{\nu}^{a}=e_{a}^{\lambda} S_{\lambda}^{\mu \nu} \partial_{[\mu} E_{\nu]}^{a}=S_{\lambda}{ }^{\mu \nu} T_{\mu \nu}^{\lambda} / 2=$ $T / 2$. Then the equation for the trace becomes

$$
2 e \partial_{\mu}\left(f^{\prime}(T) E T^{\mu}\right)+f^{\prime}(T) T-2 f(T)=-\kappa \mathcal{T},
$$

i.e.,

$2 T^{\mu} \partial_{\mu} f^{\prime}(T)+2 f^{\prime}(T) e \partial_{\mu}\left(E T^{\mu}\right)+f^{\prime}(T) T-2 f(T)=-\kappa \mathcal{T}$.

From Eq. (23), $2 e \partial_{\mu}\left(E T^{\mu}\right)$ can be substituted with $R+T$. By writing Eq. (45) in terms of $\phi$ one gets

$$
2 T^{\mu} \partial_{\mu} \phi+2 V(\phi)+\phi R=-\kappa \mathcal{T} .
$$

In TEGR it is $\phi=1$ and $V(\phi)=0$, and the former equation just implies $R=-\kappa \mathcal{T}$ [cf. Eq. (27)]. So, in principle, Eq. (47) could be interpreted as describing the

\footnotetext{
${ }^{2}$ These equations have a nontrivial antisymmetric part

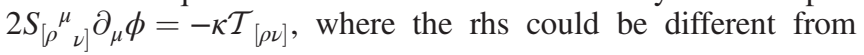
zero if the matter were coupled in a nontrivial way to the tetrad field. In Ref. [52] the lhs has been obtained from another procedure, where the action is varied with respect to an "inertial" spin connection. This procedure has been used to find the spin connection in cosmological and spherically symmetric spacetimes in [53].
}

propagation of a mode that was not present in TEGR, like Eq. (3) in $f(R)$ gravity. The existence of an extra d.o.f. is connected to the loss of a gauge symmetry. In fact, TEGR dynamics is invariant under local Lorentz transformations of the tetrad because the Lagrangian $T$ in (19) differs from $R$ in a four-divergence [see Eq. (23)], $R$ being invariant under local Lorentz transformations of the tetrad field since it only depends on the metric. Thus TEGR is not a dynamical theory for the tetrad but for the metric. Instead the gauge symmetries of the action (28) are reduced to those of $T$, which does not remain invariant under a general local Lorentz transformation. Such a remnant gauge symmetry of $f(T)$ gravity has an on-shell character. Notice that the invariance of $T$ implies the invariance of $\phi$ in Eq. (42). On the other hand, according to Eq. (23), the invariance of $T$ is equivalent to the invariance of the fourdivergence $e \partial_{\mu}\left(E T^{\mu}\right)$. Under local Lorentz transformations of the tetrad field, $e \partial_{\mu}\left(E T^{\mu}\right)$ transforms as

$$
\begin{aligned}
e \partial_{\mu}\left(E T^{\mu}\right) \rightarrow & e \partial_{\mu}\left(E g^{\mu \nu} \Lambda_{a^{\prime}}^{a} e_{a}^{\rho}\left(\partial_{\rho}\left(\Lambda_{b}^{a^{\prime}} E_{\nu}^{b}\right)-\partial_{\nu}\left(\Lambda_{b}^{a^{\prime}} E_{\rho}^{b}\right)\right)\right) \\
= & e \partial_{\mu}\left(E T^{\mu}\right) \\
& +e \partial_{\mu}\left(E g^{\mu \nu}\left(E_{\nu}^{b} e_{a^{\prime}}^{\rho} \partial_{\rho} \Lambda_{b}^{a^{\prime}}-\Lambda_{a^{\prime}}^{a} \partial_{\nu} \Lambda_{a}^{a^{\prime}}\right)\right)
\end{aligned}
$$

for infinitesimal transformations

$$
\Lambda^{a^{\prime}}{ }_{b}=\delta^{a^{\prime}}{ }_{b}-\frac{1}{2} \sigma^{g h}(x)\left(M_{g h}\right)^{a^{\prime}}{ }_{b}+O\left(\sigma^{2}\right),
$$

where the generators $M_{g h}$ are the traceless matrices $\left(M_{g h}\right)^{a^{\prime}}{ }_{b}=\delta_{g}^{a^{\prime}} \eta_{h b}-\delta_{h}^{a^{\prime}} \eta_{g b}$, it results

$$
\begin{aligned}
e \partial_{\mu}\left(E T^{\mu}\right) \rightarrow & e \partial_{\mu}\left(E T^{\mu}\right)-\frac{1}{2} e \partial_{\mu}\left(E g ^ { \mu \nu } E _ { \nu } ^ { b } \left(e_{g}^{\rho} \eta_{h b}\right.\right. \\
& \left.\left.-e_{h}^{\rho} \eta_{g b}\right) \partial_{\rho} \sigma^{g h}\right)+O\left(\sigma^{2}\right) .
\end{aligned}
$$

By replacing $g^{\mu \nu} E_{\nu}^{b} \eta_{h b}=e_{h}^{\mu}$, and noticing that $\left(e_{g}^{\rho} e_{h}^{\mu}-e_{g}^{\mu} e_{h}^{\rho}\right) \partial_{\mu} \partial_{\rho} \sigma^{g h}=0$, we obtain that the remnant gauge symmetry is generated by those Lorentz transformations that fulfill [cf. Eq. (32) in Ref. [34]]

$$
\partial_{\mu}\left(E\left(e_{g}^{\rho} e_{h}^{\mu}-e_{g}^{\mu} e_{h}^{\rho}\right)\right) \partial_{\rho} \sigma^{g h}=0 .
$$

To finish this subsection, we will notice that Eq. (47) could admit solutions $\phi=$ constant [i.e., $T=$ const, according to Eq. (42)]. In such a case Eqs. (43) look like TEGR equations with peculiar gravitational and cosmological constants. Remarkably more than one solution of this type could be found for the same potential $V(\phi)$ [i.e., for the same function $f(T)][40]$.

\section{B. Einstein frame}

Let us examine whether the issue of the number of d.o.f. in $f(T)$ gravity can be better understood in the so-called Einstein frame. We prevent the reader from possible 
confusion clarifying that in this work we will refer to the "Einstein frame" representation of $f(T)$ gravity as the action that results from applying a conformal transformation to the Jordan frame action of $f(T)$ gravity. This is a somehow forced terminology, since it is common knowledge that the outcome of such conformal transformation is not the TEGR action plus a scalar field, as it would be desired given that a similar fact happens in $f(R)$ gravity. We follow the standard procedure and start from the Jordan frame action (44), which could be regarded as the particular case $\omega_{B D}=0$ of a sort of Brans-Dicke teleparallel action of the form

$$
\begin{aligned}
S_{B D}= & \frac{1}{2 \kappa} \int d^{4} x E\left[\phi T+\omega_{B D} \phi^{-1} g^{\mu \nu} \partial_{\mu} \phi \partial_{\nu} \phi-V(\phi)\right] \\
& +S_{\text {matter }} .
\end{aligned}
$$

Now we will consider a change of dynamical variables by performing a local conformal transformation of the tetrad:

$$
\hat{E}_{\mu}^{a}=\Omega(x) E_{\mu}^{a}, \quad \hat{e}_{a}^{\mu}=\Omega^{-1}(x) e_{a}^{\mu},
$$

so it is $\hat{E}=\Omega^{4} E$ and $\hat{e}=\Omega^{-4} e$. It is well known that the action (44) will transform as [15,35]

$$
\begin{aligned}
S= & \frac{1}{2 \kappa} \int d^{4} x \hat{E}\left[\phi \left(\Omega^{-2} \hat{T}-4 \Omega^{-3} \hat{T}^{\mu} \partial_{\mu} \Omega\right.\right. \\
& \left.\left.-6 \Omega^{-4} \hat{g}^{\mu \nu} \partial_{\mu} \Omega \partial_{\nu} \Omega\right)-\Omega^{-4} V(\phi)\right]
\end{aligned}
$$

(we can disregard $S_{\text {matter }}$ since we are only interested in the number of gravitational d.o.f.). We will choose the conformal factor $\Omega$ to have a minimal coupling between $\phi$ and the torsion scalar $T$. The choice $\phi=\Omega^{2}$ converts the action (44) into

$$
\begin{aligned}
S= & \frac{1}{2 \kappa} \int d^{4} x \hat{E}\left[\hat{T}-2 \phi^{-1} \hat{T}^{\mu} \partial_{\mu} \phi\right. \\
& \left.-\frac{3}{2} \phi^{-2} \hat{g}^{\mu \nu} \partial_{\mu} \phi \partial_{\nu} \phi-\phi^{-2} V(\phi)\right] .
\end{aligned}
$$

In this action it would be desirable to have the canonical form for the kinetic term; therefore it is convenient to redefine the scalar field to a new field $\psi$ such that

$$
\psi=\sqrt{3} \ln \phi,
$$

and the action (55) is rewritten as

$S=\frac{1}{2 \kappa} \int d^{4} x \hat{E}\left[\hat{T}-\frac{2}{\sqrt{3}} \partial_{\mu} \psi \hat{T}^{\mu}-\frac{1}{2} \hat{g}^{\mu \nu} \partial_{\mu} \psi \partial_{\nu} \psi-U(\psi)\right]$,

where the potential is $U(\psi)=\phi^{-2} V(\phi)$. After an integration by parts the action reads

$$
\begin{aligned}
S= & \frac{1}{2 \kappa} \int d^{4} x \hat{E}\left[\hat{T}+\frac{2}{\sqrt{3}} \psi \hat{e} \partial_{\mu}\left(\hat{E} \hat{T}^{\mu}\right)\right. \\
& \left.-\frac{1}{2} \hat{g}^{\mu \nu} \partial_{\mu} \psi \partial_{\nu} \psi-U(\psi)\right] .
\end{aligned}
$$

This action exhibits an ordinary teleparallel theory together with a phantom scalar field. However there is also an annoying term that (nonminimally) couples the ordinary gravity to the scalar field. So, the Einstein frame in $f(T)$ gravity is unable to cleanly separate the extra d.o.f., as it effectively happens in $f(R)$ gravity.

Although we have already shown a rather strong evidence in favor of an extra d.o.f. in $f(T)$ gravity, the issue can be definitely solved within the formalism for constrained Hamiltonian systems, as it was done in a recent publication [42]. We can gain some insight into this formalism by looking at how it works in a simple toy model, as we are going to show in the next section.

\section{MODIFYING A MECHANICAL SYSTEM WITH ROTATIONAL PSEUDOINVARIANCE}

\section{A. Pseudoinvariant rotational Lagrangian}

Some features of $f(T)$ gravity can be mimicked by deforming the mechanical Lagrangian

$$
L=\frac{1}{2}\left(\frac{\dot{z}}{z}+\frac{\dot{\bar{z}}}{\bar{z}}\right)^{2}+A \frac{\dot{z}}{z}+\bar{A} \frac{\dot{\bar{z}}}{\bar{z}}-U(z \bar{z}),
$$

where $z$ and $\bar{z}$ are complex conjugate canonical variables and $A$ and $\bar{A}$ are complex conjugate constants. This Lagrangian can be rewritten as

$L=\frac{1}{2}\left(\frac{d}{d t} \ln (z \bar{z})\right)^{2}+\frac{d}{d t}(A \ln z+\bar{A} \ln \bar{z})-U(z \bar{z})$,

so it just provides a dynamics to the combination $z \bar{z}$, since the total derivative term is irrelevant in Lagrange equations. This means that the evolution of the phase $z /|z|$ is not determined by Lagrange equations. We can notice this fact also at the level of the symmetries of the Lagrangian, which is pseudoinvariant under local rotations:

$z \rightarrow e^{i \alpha(t)} z, \quad \bar{z} \rightarrow e^{-i \alpha(t)} \bar{z} \Rightarrow L \rightarrow L+i \dot{\alpha}(A-\bar{A})$.

We can recognize some features that resemble the TEGR theory. In fact, the TEGR Lagrangian is pseudoinvariant under local Lorentz transformations of the tetrad; so it can only govern the dynamics of the metric, but it is unable to determine the "orientation" of the tetrad. The analogy is not complete because the boundary term in this toy model just contain first derivatives of the canonical variables, differing from the boundary term in Eq. (23) which is second order in derivatives of the tetrad. 
Now let us pass to the Hamiltonian formalism and look for the constraint algebra. The canonical momenta are defined as

$p_{z} \equiv \frac{\partial L}{\partial \dot{z}}=\frac{1}{z}\left(\frac{\dot{z}}{z}+\frac{\dot{\bar{z}}}{\bar{z}}+A\right), \quad p_{\bar{z}} \equiv \frac{\partial L}{\partial \dot{\bar{z}}}=\frac{1}{\bar{z}}\left(\frac{\dot{z}}{z}+\frac{\dot{\bar{z}}}{\overline{\bar{z}}}+\bar{A}\right)$,

from which it is easily seen the primary constraint

$$
G^{(1)} \equiv z p_{z}-A-\bar{z} p_{\bar{z}}+\bar{A} \approx 0,
$$

which fulfills

$$
\left\{G^{(1)}, z \bar{z}\right\}=0 .
$$

The canonical Hamiltonian is

$$
\begin{aligned}
H & =\dot{z} p_{z}+\dot{\bar{z}} p_{\bar{z}}-L=\frac{1}{2}\left(\frac{\dot{z}}{z}+\frac{\dot{\bar{z}}}{\bar{z}}\right)^{2}+U(z \bar{z}) \\
& =\frac{1}{8}\left(z p_{z}-A+\bar{z} p_{\bar{z}}-\bar{A}\right)^{2}+U(z \bar{z}),
\end{aligned}
$$

while the primary Hamiltonian results

$H_{p}=\frac{1}{8}\left(z p_{z}-A+\bar{z} p_{\bar{z}}-\bar{A}\right)^{2}+U(z \bar{z})+u(t) G^{(1)}$,

where $u(t)$ is a Lagrange multiplier. We verify that $G^{(1)}$ evolves without leaving the constraint surface:

$$
\begin{aligned}
\dot{G}^{(1)}= & \left\{G^{(1)}, H_{p}\right\}=\frac{1}{4}\left(z p_{z}-A+\bar{z} p_{\bar{z}}-\bar{A}\right) \\
& \times\left\{G^{(1)}, z p_{z}-A+\bar{z} p_{\bar{z}}-\bar{A}\right\}=0 .
\end{aligned}
$$

Therefore, no secondary constraints appear. There is a unique first-class constraint; so one d.o.f. is removed, and the system is left with just one genuine d.o.f. [70]. Equation (64) reveals that $z \bar{z}$ is the gauge invariant (observable) associated to the physical d.o.f.

\section{B. Modified pseudoinvariant rotational Lagrangian}

Let us deform the theory by replacing the pseudoinvariant Lagrangian with a function of itself:

$$
\mathcal{L}=f(L)
$$

This new theory is dynamically equivalent to the one governed by the Jordan frame Lagrangian that includes an additional dynamical variable $\phi$ :

$$
\mathcal{L}=\phi L-V(\phi) .
$$

In fact, the dynamical equation for $\phi$ is

$$
L=V^{\prime}(\phi) \text {. }
$$

So, the dynamics says that $\mathcal{L}$ in Eq. (69) is the Legendre transform of $V(\phi)$; therefore, $\mathcal{L}$ is a function $f(L)$ (each choice of $V$ equals a choice of $f$ ). The canonical momenta are

$$
G_{\pi}^{(1)} \equiv \pi=\frac{\partial \mathcal{L}}{\partial \dot{\phi}} \approx 0
$$

$p_{z}=\frac{\phi}{z}\left(\frac{\dot{z}}{z}+\frac{\dot{\bar{z}}}{\bar{z}}+A\right), \quad p_{\bar{z}}=\frac{\phi}{\bar{z}}\left(\frac{\dot{z}}{z}+\frac{\dot{\bar{z}}}{\bar{z}}+\bar{A}\right) ;$

thus one gets the constraint

$$
G^{(1)} \equiv z p_{z}-A \phi-\bar{z} p_{\bar{z}}+\bar{A} \phi \approx 0 .
$$

Then the modified system has two noncommuting primary constraints:

$$
\left\{G^{(1)}, G_{\pi}^{(1)}\right\}=-A+\bar{A} .
$$

The canonical Hamiltonian is

$$
\begin{aligned}
\mathcal{H}= & \dot{z} p_{z}+\dot{\bar{z}} p_{\bar{z}}-\mathcal{L}=\frac{1}{8 \phi}\left(z p_{z}-\phi A+\bar{z} p_{\bar{z}}-\phi \bar{A}\right)^{2} \\
& +\phi U(z \bar{z})+V(\phi),
\end{aligned}
$$

and the primary Hamiltonian is

$$
\mathcal{H}_{p}=\mathcal{H}+u^{\pi}(t) G_{\pi}^{(1)}+u(t) G^{(1)},
$$

where $u^{\pi}$ and $u$ are Lagrange multipliers. We must evaluate the evolution of the primary constraints to look for secondary constraints:

$$
\begin{aligned}
\dot{G}_{\pi}^{(1)}= & \left\{G_{\pi}^{(1)}, \mathcal{H}_{p}\right\}=\left\{\pi, \mathcal{H}_{p}\right\} \\
= & \frac{\left(z p_{z}+\bar{z} p_{\bar{z}}\right)^{2}}{8 \phi^{2}}-\frac{1}{8}(A+\bar{A})^{2}-U(z \bar{z})-V^{\prime}(\phi) \\
& +u(t)(A-\bar{A}) \\
= & L-\frac{1}{2}(A-\bar{A})\left(\frac{\dot{z}}{z}-\frac{\dot{\bar{z}}}{\bar{z}}\right)-V^{\prime}(\phi)+u(t)(A-\bar{A}),
\end{aligned}
$$

$$
\dot{G}^{(1)}=\left\{G^{(1)}, \mathcal{H}_{p}\right\}=-u^{\pi}(A-\bar{A}) .
$$

Thus, the consistency of the constraints with the evolution can be obtained by choosing the Lagrange multipliers as

$u^{\pi}=0, \quad u(t)=\frac{L(t)-V^{\prime}(\phi(t))}{A-\bar{A}}-\frac{1}{2}\left(\frac{\dot{z}(t)}{z(t)}-\frac{\dot{\bar{z}}(t)}{\bar{z}(t)}\right)$. 
Therefore the system has no secondary constraints; the only constraints $G^{(1)}$ and $G_{\pi}^{(1)}$ are second class [see Eq. (74)]. So, they remove only one d.o.f. [70]; there are two genuine d.o.f. among the variables $(z, \bar{z}, \phi)$. Notice that, differing from $f(T)$ gravity, no gauge freedom is left in this system since both Lagrange multipliers have been fixed; so the primary Hamiltonian completely determines the evolution of the variables. In particular, the evolution of $\phi$ is frozen:

$$
\dot{\phi}=\left\{\phi, \mathcal{H}_{p}\right\}=u^{\pi}=0 .
$$

Coming back to the Lagrange equations, let us use for $L$ the form (60); then by varying $\mathcal{L}$ with respect to $z \bar{z}$ and $z$ one obtains

$$
\frac{d}{d t}\left(\phi \frac{d}{d t} \ln (z \bar{z})\right)=-\phi U^{\prime}(z \bar{z}), \quad \frac{d}{d t}(\phi A)=0 .
$$

So, we retrieve the result that $\phi$ is constant. Besides $z \bar{z}$ obeys the same dynamical equation of the nondeformed theory. In particular the magnitude

$$
H=\frac{1}{2}\left(\frac{d}{d t} \ln (z \bar{z})\right)^{2}+U(z \bar{z})
$$

is a constant of motion. Since $\phi$ is constant, then the dynamical equation for $\phi$, i.e., Eq. (70), implies that $L$ is constant. From Eqs. (70), (59) and (82) we obtain that the evolution of the phase of $z$ is determined by the dynamics through the first-order equation

$$
\begin{aligned}
\frac{d}{d t}(A \ln z+\bar{A} \ln \bar{z}) & =-\frac{1}{2}\left(\frac{d}{d t} \ln (z \bar{z})\right)^{2}+U(z \bar{z})+V^{\prime}(\phi) \\
& =2 U(z \bar{z})+V^{\prime}(\phi)-H
\end{aligned}
$$

At the initial conditions, the choice of $\phi$ is the way one chooses the initial velocity of the phase. Due to the remaining global rotational symmetry, the initial value of the phase is irrelevant. This equation can be also obtained in the Hamiltonian formalism by computing the Poisson bracket between $A \ln z+\bar{A} \ln \bar{z}$ and $\mathcal{H}_{p}$ and then substituting the momenta and the Lagrange multipliers with (72) and (79).

In sum, the system described by the Lagrangian (69) has two d.o.f.: one of them is $|z|^{2}=z \bar{z}$ whose dynamics does not differ from the one described by the Lagrangian (60); in both cases we arrive to the conserved quantity $H$ in Eq. (82). Once the evolution of $|z|$ is determined by the choices of the initial value $\left|z\left(t_{o}\right)\right|$ and the constant of motion $H$, Eq. (83) determines the evolution of the phase of $z$, which is the remaining d.o.f. In this equation, the value of the constant $\phi$ is connected to the initial value of the derivative of the phase of $z$; there is no other physics associated with $\phi$ over and above the one related to the phase of $z$.
In analogy with $f(T)$ gravity, $\phi$ could be then regarded as a variable carrying information about the orientation of the tetrad, which would be partially determined by the dynamical equations.

\section{HAMILTONIAN FORMALISM AND FRAME DEPENDENCE}

The Hamiltonian formalism for $f(T)$ gravity, starting from the (scalar-torsion) Jordan frame formulation of the theory, was presented in full detail in [42]. There are several unusual features of this physical system that deserves a comment. In most Hamiltonian constrained systems the identification of secondary constraints is a rather trivial enterprise, and there is no need to calculate the left and right null eigenvectors of the matrix of Poisson brackets among constraints. However, in the Hamiltonian formalism for $f(T)$ gravity we have to pay close attention to this point, which makes the theory an atypical example of a constrained Hamiltonian system.

After following the Dirac-Bergmann algorithm for $f(T)$ gravity one is led to a set of first-class $\Phi_{I}$ and second-class constraints $\chi_{A}$ organized in the following way (see definitions at [42]):

(i) $n$ first-class constraints $\Phi_{c}=G_{c}^{(1)}$ coming from the absence of $\dot{E}_{0}^{c}$ in the Lagrangian,

(ii) $n-1$ first-class constraints $\Phi_{i}=G_{i}^{(2)}$ associated with the spatial diffeomorphisms (supermomentum constraints),

(iii) $\frac{n(n-1)}{2}-1$ first-class constraints $\Phi_{a b}=\tilde{G}_{a b}^{(1)}$ associated with the Lorentz algebra,

(iv) one first-class constraint $\Phi_{0}=\tilde{G}_{0}^{(2)}$ associated with the temporal diffeomorphism (super-Hamiltonian constraint), and

(v) two second-class constraints: $\chi_{\pi}=G_{\pi}^{(1)}$, coming from the absence of $\dot{\phi}$ in the Lagrangian, and $\chi=$ $\alpha^{a b} \tilde{G}_{a b}^{(1)}$ associated with the Lorentz algebra.

This classification is obtained after a redefinition of the original Lorentz constraints that maximizes the number of vanishing Poisson brackets in the algebra of constraints. In fact, in terms of the original constraints, the nonvanishing Poisson brackets involve the Lorentz constraints and the super-Hamiltonian constraint; they are

$$
\begin{aligned}
& \left\{G_{a b}^{(1)}, G_{\pi}^{(1)}\right\} \equiv F_{a b}, \\
& \left\{G_{0}^{(2)}, G_{\pi}^{(1)}\right\} \equiv F_{\phi} .
\end{aligned}
$$

Since $F_{a b}$ and $F_{\phi}$ are functions of the canonical coordinates and momenta, they could vanish at particular points of the phase space. So, let us suppose that $F_{01}$ is not zero in some neighborhood of the constraint surface. Then we can change the basis of the constraint algebra by combining the original Lorentz constraints $G_{a b}^{(1)}$ in the following way: 


$$
\begin{aligned}
\tilde{G}_{01}^{(1)} & =G_{01}^{(1)}, \\
\tilde{G}_{02}^{(1)} & =F_{01} G_{02}^{(1)}-F_{02} G_{01}^{(1)}, \\
& \vdots \\
\tilde{G}_{(n-2)(n-1)}^{(1)} & =F_{01} G_{(n-2)(n-1)}^{(1)}-F_{(n-2)(n-1)} G_{01}^{(1)} .
\end{aligned}
$$

Besides, we replace the super-Hamiltonian constraint $G_{0}^{(2)}$ with

$$
\tilde{G}_{0}^{(2)}=F_{01} G_{0}^{(2)}-F_{\phi} G_{01}^{(1)} .
$$

Thus the only nonvanishing Poisson brackets will be

$$
\left\{\tilde{G}_{01}^{(1)}, G_{\pi}^{(1)}\right\} \equiv F_{01}
$$

In this way we have two second-class constraints $\chi_{A}$, the remaining constraints being first class. The matrix $\Delta_{A B}=\left\{\chi_{A}, \chi_{B}\right\}$,

$\Delta_{A B}=\left(\begin{array}{ll}\left\{G_{\pi}^{(1)}, G_{\pi}^{(1)}\right\} & \left\{\tilde{G}_{01}^{(1)}, G_{\pi}^{(1)}\right\} \\ \left\{G_{\pi}^{(1)}, \tilde{G}_{01}^{(1)}\right\} & \left\{\tilde{G}_{01}^{(1)}, \tilde{G}_{01}^{(1)}\right\}\end{array}\right)=\left(\begin{array}{cc}0 & F_{01} \\ -F_{01} & 0\end{array}\right)$,

is a $2 \times 2$ invertible matrix. The choice that $\tilde{G}_{01}^{(1)}$ be the odd second-class Lorentz constraint is completely arbitrary and any linear combination of the form

$$
\chi=\alpha^{a b} G_{a b}^{(1)}
$$

can be chosen to be the second-class one (but $\alpha^{a b} F_{a b}$ must be different from zero). This is because the DiracBergmann algorithm does not determine a particular linear combination to be second class. However the arbitrariness of the choice does not affect the conclusions.

The equations assuring the consistency of the evolution of the first-class constraints result to be trivial, while the ones for the second-class constraints $\chi_{A}=\left(G_{\pi}^{(1)}, \tilde{G}_{01}^{(1)}\right)$,

$$
\left\{\chi_{A}, H_{c}\right\}+\Delta_{A B} u^{B} \stackrel{!}{\approx} 0
$$

can be satisfied by properly choosing the Lagrange multipliers $u^{\pi}$ and $\tilde{u}^{01}$ :

$$
u^{\pi}=0, \quad F_{01} \tilde{u}^{01}=F_{\phi},
$$

which is comparable to Eq. (79) in Sec. V. Therefore, when passing from TEGR to $f(T)$ gravity as described in the Jordan frame, one of the first-class constraints associated with the Lorentz algebra becomes a second-class constraint paired with $G_{\pi}^{(1)}$. As was shown in the toy model of the previous section, this fact amounts to the retrieval of a d.o.f. that took part in the gauge freedom of the TEGR theory. Concretely, the invariance under local Lorentz transformations is partially lost since a linear combination of their generators is no longer a gauge transformation. In other words, some feature of the tetrad orientation has passed to be governed by the dynamics and now forms part of the set of genuine d.o.f. together with the metric d.o.f.

For defining the set of first-class Lorentz constraints (85) we had to assume that at least one of the $F_{a b}$ is nonvanishing. However, there could be points on the constraint surface where all the $F_{a b}$ vanishes. In this case, the consistency of the Dirac-Bergmann algorithm could impose two different outcomes: $F_{\phi}$ also vanishes identically and the theory has the same d.o.f. as TEGR, or $F_{\phi}$ is a constraint and the algorithm continues. Although, for obtaining a meaningful number of d.o.f., the algorithm is not allowed to extend too much, as we will argue next. Let us reasonably assume that neither $G_{\mu}^{(2)}$ nor $G_{c}^{(1)}$ become second class and thus they remove $2 n$ d.o.f. But $X$ of the $n(n-1) / 2$ Lorentz constraints could still become second class due to the pairing with the same amount of secondclass constraints. Besides, there could appear additional $Y_{1}$ first-class constraints (f.c.c.) and $Y_{2}$ second-class constraints (s.c.c.). The counting of d.o.f. would go in the following way:

number of d.o.f. $=n^{2}+1-$ (number of f.c.c.)

$$
\begin{aligned}
& -\frac{1}{2}(\text { number of s.c.c. }) \\
= & n^{2}+1-\left(2 n+\frac{n}{2}(n-1)-X+Y_{1}\right) \\
& -\frac{1}{2}\left(2 X+Y_{2}\right) \\
= & \frac{n(n-3)}{2}+1-Y_{1}-\frac{1}{2} Y_{2} .
\end{aligned}
$$

The case of $f(T)$ gravity falls in the category $X=1$, since there is only one constraint $G_{\pi}^{(1)}$ that pairs up with one Lorentz constraint to become second class. If we are in a particular point of the phase space where all the $F_{a b}$ are zero, the Poisson bracket among $G_{\pi}^{(1)}$ and $G_{a b}^{(1)}$ would vanish on shell, but some of the $G_{a b}^{(1)}$ could still become second class due to the appearance of new constraints represented by $X$. In this case the algorithm would require $F_{\phi}$ to weakly vanish on shell and become a constraint. If it were first class, then $Y_{1}=1, Y_{2}=0$; thus Eq. (92) would reproduce the number of d.o.f. of TEGR. Instead, if both $F_{\phi}$ and $G_{\pi}^{(1)}$ are second class and they originate at least one new first- or two second-class constraints, the counting of d.o.f. will give a meaningless answer: less d.o.f. than TEGR. This, of course, is an unacceptable result, and 
the only reasonable possibility is that the pair $F_{\phi}, G_{\pi}^{(1)}$ is second class and removes one d.o.f. to give the TEGR result. In this case, the time stability of $F_{\phi}$ should be proved calculating its Poisson algebra with the remaining constraints; however it is a very involved calculation which may require an improved Hamiltonian formalism.

Finally, it is worth mentioning a puzzling feature in the Hamiltonian constraint of Eq. (86), since the second term of this expression may have a cubic dependence on the canonical momenta. This is easily seen because $G_{01}^{(1)}$ is linear in the momenta, but $F_{\phi}$ depends on the torsion scalar and is therefore quadratic in the momenta. The cubic dependence of $\tilde{G}_{0}^{(2)}$ is the price to be paid for getting a first-class Hamiltonian constraint. This fact can be traced to the pseudoinvariance of the TEGR Lagrangian, which implies that the $f(T)$ action breaks the local Lorentz invariance. This is an unusual feature for a Hamiltonian system, and it could lead to instabilities since the Hamiltonian could be unbounded from below $[71,72]$.

\section{HAMILTONIAN APPROACH IN THE TELEPARALLEL EINSTEIN FRAME}

Since the conformal transformation that leads from the Jordan frame to the Einstein frame is a canonical transformation, one should expect the same number of d.o.f. in both the Jordan and Einstein frames. Nonetheless this mere verification could throw light on the issue of the meaning of the extra d.o.f. However this exercise will indeed reveal the intricate Hamiltonian structure of $f(T)$ gravity in the Einstein frame. Let us start from a gravitational theory whose action is

$$
\begin{aligned}
S\left[E_{\mu}^{a}, \psi\right]= & \frac{1}{2 \kappa} \int d^{4} x E\left(T+\frac{2}{\sqrt{3}} \psi B\right. \\
& \left.-\frac{1}{2} g^{\mu \nu} \nabla_{\mu} \psi \nabla_{\nu} \psi+U(\psi)\right) .
\end{aligned}
$$

We get rid of the hatted fields in the action and start working directly under the assumption that the teleparallel Einstein frame is our theory for describing the gravitational phenomena. In this formulation it is simple to see that the Hamiltonian procedure is not applicable, since the term $B$, as we have written it in Eq. (37), has an explicit dependence on second-order time derivatives on the tetrad field. In this case we need to resort to Ostrogradsky's choice for canonical variables and momenta. It is well known that Lagrangians having higher-order time derivatives might suffer from the Ostrogradsky instability [71,72], although as we will prove later, in our case we can get rid of this obstruction by means of an integration by parts. However, motivated by a deeper understanding in this subject, it will be interesting to first explore this approach. Ostrogradsky's definitions of canonical momenta are given by
$\Pi_{a}^{\mu}=\frac{\partial L}{\partial\left(\partial_{0} E_{\mu}^{a}\right)}-\partial_{0} \frac{\partial L}{\partial\left(\partial_{0} \partial_{0} E_{\mu}^{a}\right)}, \quad \mathcal{P}_{a}^{\mu}=\frac{\partial L}{\partial\left(\partial_{0} \partial_{0} E_{\mu}^{a}\right)}$,

that are associated to the canonical variables $E_{\mu}^{a}$ and $\mathcal{E}_{\mu}^{a} \equiv \partial_{0} E_{\mu}^{a}$, respectively. Within the premetric formalism developed in Sec. III, they can be easily computed; the result is

$$
\begin{aligned}
\Pi_{a}^{\mu}= & E \partial_{\rho} E_{\lambda}^{b} e_{c}^{0} e_{e}^{\mu} e_{d}^{\rho} e_{f}^{\lambda}\left(M_{a b}^{c e d f}+\frac{2}{\sqrt{3}} \psi B_{a b}{ }^{c e d f}\right) \\
& -\frac{2}{\sqrt{3}} \partial_{0}\left[\psi E e_{b}^{0} e_{c}^{\mu} e_{d}^{0}\right]\left(\delta_{a}^{d} \eta^{b c}-\delta_{a}^{c} \eta^{b d}\right)
\end{aligned}
$$

and

$$
\mathcal{P}_{a}^{\mu}=\frac{2}{\sqrt{3}} \psi E e_{b}^{0} e_{c}^{\mu} e_{d}^{0}\left(\delta_{a}^{d} \eta^{b c}-\delta_{a}^{c} \eta^{b d}\right)
$$

There is also a canonical momentum associated with the scalar field $\psi$, given by

$$
\pi=\frac{\partial L}{\partial\left(\partial_{0} \psi\right)}=-E g^{0 \nu} \partial_{\nu} \psi
$$

It is clear that there appear $n$ primary constraints $\mathcal{P}_{a}^{0} \approx 0$. Besides, (95) can be rewritten as a primary constraint using (97), which is

$$
\begin{aligned}
\Pi_{a}^{\mu}= & E \mathcal{E}_{\lambda}^{b} e_{e}^{\mu} e_{f}^{\lambda} \tilde{C}_{a b}{ }^{e f} \\
& +E \partial_{i} E_{\lambda}^{b} e_{c}^{0} e_{e}^{\mu} e_{d}^{i} e_{f}^{\lambda}\left(M_{a b}{ }^{c e d f}+\frac{2}{\sqrt{3}} \psi B_{a b}{ }^{c e d f}\right) \\
& +\frac{2}{\sqrt{3}}\left(\frac{e}{g^{00}} \pi+\frac{g^{00}}{g^{0 i}} \partial_{i} \psi\right) E\left[e_{a}^{0} g^{0 \mu}-e_{a}^{\mu} g^{00}\right],
\end{aligned}
$$

where

$$
\begin{aligned}
\tilde{C}_{a b}{ }^{e f}= & e_{c}^{0} e_{d}^{0}\left(M_{a b}{ }^{c e d f}+\frac{2}{\sqrt{3}} \psi B_{a b}{ }^{c e d f}-\frac{4}{\sqrt{3}} \psi\left(\eta^{c f} \delta_{a}^{[e} \delta_{b}^{d]}\right.\right. \\
& \left.\left.+\eta^{c d} \delta_{a}^{[f} \delta_{b}^{e]}+\eta^{e c} \delta_{a}^{[d} \delta_{b}^{f]}+\delta_{b}^{d} \eta^{f[c} \delta_{a}^{e]}\right)\right)
\end{aligned}
$$

As depicted in [72], Eqs. (95)-(97) should be used to write the canonical Hamiltonian

$$
H_{c}=\Pi_{a}^{\mu} \mathcal{E}_{\mu}^{a}+\mathcal{P}_{a}^{\mu} \partial_{0} \mathcal{E}_{\mu}^{a}+\pi \partial_{0} \psi-L
$$

in terms of the canonical variables $\left(E_{\mu}^{a}, \Pi_{a}^{\mu}, \mathcal{E}_{\mu}^{a}, \mathcal{P}_{a}^{\mu}, \psi, \pi\right)$ and their spatial derivatives. According to the formalism, the acceleration $\mathcal{A}_{\mu}^{a}=\partial_{0} \partial_{0} E_{\mu}^{a}$ should be solved as a function of the canonical variables from the second equation in Eq. (94); thus, $\Pi_{a}^{\mu}$ will not enter in the 
expression for $\mathcal{A}_{\mu}^{a}$. Therefore, $H_{c}$ will depend on $\Pi_{a}^{\mu}$ only through the first term of Eq. (100), which is a linear in $\Pi_{a}^{\mu}$ (in this higher-order formalism, $\mathcal{E}_{\mu}^{a}$ is a canonical variable). This means that $H_{c}$ is unbounded from below and we might be in the presence of some sort of instability, although briefly we will see that we can circumvent this issue resorting to an equivalent representation.

However, the Lagrangian in Eq. (93) is linear in the acceleration $\mathcal{A}_{\mu}^{a}=\partial_{0} \partial_{0} E_{\mu}^{a}=\partial_{0} \mathcal{E}_{\mu}^{a}$. Therefore the definition of $\mathcal{P}_{a}^{\mu}$ does not allow one to solve the acceleration in terms of the canonical variables but leads to a constraint [cf. Eq. (96)]. Thus, the acceleration will remain as a Lagrange multiplier in the canonical Hamiltonian (100), which will be subsumed in the respective Lagrange multiplier of the primary Hamiltonian.

Ostrogradsky's procedure can be avoided by performing an integration by parts in (93); thus we will work with the action

$$
\begin{aligned}
S\left[E_{\mu}^{a}, \psi\right]= & \frac{1}{2 \kappa} \int d^{4} x E\left(T-\frac{2}{\sqrt{3}} T^{\mu} \partial_{\mu} \psi\right. \\
& \left.-\frac{1}{2} g^{\mu \nu} \nabla_{\mu} \psi \nabla_{\nu} \psi+U(\psi)\right) .
\end{aligned}
$$

In this case, the canonical momenta associated with the tetrad field results

$$
\begin{aligned}
\Pi_{a}^{\mu}= & E \partial_{\rho} E_{\lambda}^{b} e_{c}^{0} e_{e}^{\mu} e_{d}^{\rho} e_{f}^{\lambda} M_{a b}{ }^{c e d f} \\
& -\frac{2}{\sqrt{3}} E \partial_{\nu} \psi e_{b}^{0} e_{c}^{\nu} e_{d}^{\mu}\left[\eta^{d c} \delta_{a}^{b}-\eta^{b c} \delta_{a}^{d}\right]
\end{aligned}
$$

while the canonical momentum for the scalar field is

$\pi=-E g^{0 \nu} \partial_{\nu} \psi-\frac{2}{\sqrt{3}} E \partial_{\rho} E_{\nu}^{a} e_{b}^{0} e_{c}^{\rho} e_{d}^{\nu}\left[\eta^{b d} \delta_{a}^{c}-\eta^{c b} \delta_{a}^{d}\right]$

It is easy to see that both canonical momenta include canonical velocities $\partial_{0} E_{\mu}^{a}$ and $\partial_{0} \psi$ in their definitions. Therefore, the procedure to write the canonical Hamiltonian in terms of the canonical variables will be more intricate than the one developed for TEGR in Ref. [66]. Let us solve for $\partial_{0} \psi$ in (103):

$$
\begin{aligned}
\partial_{0} \psi= & -\frac{e}{g^{00} \pi}-\frac{g^{0 i}}{g^{00}} \partial_{i} \psi \\
& -\frac{2}{\sqrt{3} g^{00}} \partial_{\rho} E_{\lambda}^{a} e_{b}^{0} e_{c}^{\rho} e_{d}^{\lambda}\left[\eta^{b d} \delta_{a}^{c}-\eta^{c b} \delta_{a}^{d}\right] .
\end{aligned}
$$

After replacing this result in (102) and performing some arrangements, the following expression is obtained:

$$
\begin{aligned}
E_{\mu}^{e} \Pi_{a}^{\mu}= & E \partial_{0} E_{\lambda}^{b} e_{f}^{\lambda} \tilde{C}_{a b}{ }^{e f}+E \partial_{i} E_{\lambda}^{b} e_{f}^{\lambda}\left(e_{c}^{0} e_{d}^{i} M_{a b}{ }^{c e d f}\right. \\
& \left.+\frac{4}{3 g^{00}} e_{c}^{0} e_{d}^{0} e_{g}^{0} e_{h}^{i}\left[\eta^{c e} \delta_{a}^{d}-\eta^{c d} \delta_{a}^{e}\right]\left[\eta^{g f} \delta_{b}^{h}-\eta^{h g} \delta_{b}^{f}\right]\right) \\
& +\frac{2 \pi}{\sqrt{3} g^{00}} e_{c}^{0} e_{d}^{0}\left[\eta^{c e} \delta_{a}^{d}-\eta^{c d} \delta_{a}^{e}\right] \\
& +\frac{2}{\sqrt{3}} E \partial_{i} \psi e_{d}^{0}\left(-e_{c}^{i}+e_{c}^{0} \frac{g^{0 i}}{g^{00}}\right)\left[\eta^{c e} \delta_{a}^{d}-\eta^{c d} \delta_{a}^{e}\right]
\end{aligned}
$$

where

$$
\begin{aligned}
\tilde{C}_{a b}{ }^{e f}= & e_{c}^{0} e_{d}^{0} M_{a b}{ }^{c e d f}+\frac{4}{3 g^{00}} e_{d}^{0} e_{c}^{0} e_{g}^{0} e_{h}^{0}\left[\eta^{c e} \delta_{a}^{d}-\eta^{c d} \delta_{a}^{e}\right] \\
& \times\left[\eta^{g f} \delta_{b}^{h}-\eta^{h g} \delta_{b}^{f}\right] .
\end{aligned}
$$

Apart from looking for primary constraints, the next obvious step would be to write the canonical Hamiltonian associated with (101). It can be attempted by means of the Moore-Penrose pseudoinverse method introduced in [66] in order to invert the matrix (106). For this, it would be useful to know whether its eigenvalues and eigenvectors are the same obtained for $C_{a b}{ }^{\text {ef }}$ in TEGR; however the answer is negative. Moreover, the structure of eigenvalues and eigenvectors is more complicated, and it may require to develop advanced mathematical techniques in order to find them. The fact that the matrix (106) is not the same than the one in TEGR is intriguing and puts forward the question whether the teleparallel Einstein frame can genuinely be interpreted as TEGR plus a scalar field. The answer to this interesting question calls for further research.

Finally we can attempt to obtain the primary constraints of this theory by multiplying Eq. (105) by the eigenvectors $v_{|g| e}^{a}=e_{e}^{0} \delta_{g}^{a}$ and $v_{|g h| e}{ }^{a}=\delta_{g}^{a} \eta_{h e}-\delta_{h}^{a} \eta_{g e}$. The first one gives the trivial constraint

$$
G_{g}^{(1)}=\Pi_{g}^{0} \approx 0 .
$$

For the second set of eigenvectors $v_{|g h| e}{ }^{a}$, it is easy to check that they are null eigenvectors of $\tilde{C}_{a b}{ }^{e f}$, i.e., they satisfy $v_{|g h| e}{ }^{a} \tilde{C}_{a b}{ }^{e f}=0$, as they are also for the matrix $C_{a b}{ }^{e f}$ in TEGR [66]. Using this result in (105), the following constraints are obtained:

$$
\begin{aligned}
G_{a b}^{(1)}= & 2 \eta_{e[b} \Pi_{a]}^{i} E_{i}^{e}+4 E \partial_{i} E_{j}^{b} e_{[b}^{0} e_{a}^{i} e_{c]}^{j} \\
& +\frac{2}{\sqrt{3}} \partial_{i} \psi\left(e_{a}^{i} e_{b}^{0}-e_{a}^{0} e_{b}^{i}\right) .
\end{aligned}
$$

The appearance of an extra term with respect to the TEGR case changes drastically the Lorentz algebra, as this term has a nonvanishing Poisson bracket with the $G_{c d}^{(1)}$, s. It is not difficult to prove that 


$$
\begin{aligned}
\left\{G_{a b}^{(1)}, G_{c d}^{(1)}\right\}= & \eta_{b d} G_{a c}^{(1)}+\eta_{a c} G_{b d}^{(1)}-\eta_{b c} G_{a d}^{(1)}-\eta_{a d} G_{b c}^{(1)} \\
& +\frac{4}{\sqrt{3}} \partial_{i} \psi\left[H_{a b} H_{c d}^{i}-H_{c d} H_{a b}^{i}\right],
\end{aligned}
$$

where

$$
H_{a b} \equiv e_{a}^{0} e_{0 b}-e_{b}^{0} e_{0 a}, \quad H_{c d}^{i} \equiv e_{c}^{0} e_{d}^{i}-e_{d}^{0} e_{c}^{i} .
$$

These two terms reflect the departure from Lorentz invariance, since they do not vanish in the most general case. However, unlike the teleparallel Jordan frame case, here the theory seems to present full Lorentz violation. That is, none of the Lorentz constraints seems to be first class; then one might be tempted to conclude that this leads to the counting of d.o.f. of [23]. Nevertheless, there is still another Poisson bracket $\left\{G_{g}^{(1)}, G_{a b}^{(1)}\right\}$ to be taken into account, which gives

$$
\left\{G_{g}^{(1)}, G_{a b}^{(1)}\right\}=\frac{2}{\sqrt{3}} \partial_{i} \psi e_{g}^{0}\left(e_{a}^{i} e_{b}^{0}-e_{a}^{0} e_{b}^{i}\right) .
$$

The noncommuting character of $G_{g}^{(1)}$ was expected, since gauge transformations of the tetrad in the form $\delta E_{0}^{a}=\epsilon^{a}$ are no longer symmetries of the Lagrangian due to the dependence on $\partial_{0} E_{0}^{a}$ in the coupling term proportional to $T^{\mu} \partial_{\mu} \psi$ in the action (101) (see Sec. V of [66]). Henceforth, the counting of d.o.f. in this frame requires a rigorous analysis of the time evolution of all constraints with the primary Hamiltonian, a point that needs to be investigated in future work.

\section{CONCLUSIONS}

We have analyzed the issue of the d.o.f. in $f(T)$ gravity from several perspectives. For the sake of a comparison, we first reviewed the interpretation of the additional d.o.f. in $f(R)$ gravity in both Jordan and Einstein frames. Analogously, the study of the equations of motion and its trace in $f(T)$ gravity reveals evidence of a unique additional d.o.f. in the teleparallel Jordan and Einstein frames. To mimic the essential features of $f(T)$ gravity, we have exhibited the Hamiltonian dynamics of a simple toy model with pseudorotational invariance. The nonlinear deformation of this toy model shows the appearance of a scalar d.o.f. $\phi$, whose value is connected to the initial value of the derivative of the phase of the complex canonical variable $z$. Analogously, in $f(T)$ gravity the scalar d.o.f. is connected with the proper parallelization of the spacetime.
Concerning our previous work on the extra d.o.f. of $f(T)$ gravity [42], we have emphasized that such analysis can depend on the point of the phase space under consideration. In fact, the Poisson brackets between constraints are functions of the tetrad field and their canonical momenta. Thus, there could exist a point or a neighborhood of the constraint surface where all the $F_{a b}$ 's in Eq. (84) were zero, which would dramatically change the counting of d.o.f. In that particular points the consistency of our analysis would imply that $F_{\phi}$ becomes a constraint, nonetheless if its time consistency does not generate additional constraints, the theory would have the same number of d.o.f. as TEGR. This could be suggesting that only some reference frames do manifest the extra d.o.f.; however it is still unclear the conditions that they should accomplish.

Finally, we have just introduced two possible ways of applying the Hamiltonian formalism in the teleparallel Einstein frame. In spite of appearances, the Einstein frame proves to be rather more involved than the Jordan frame. This is because the term coupling the vector part of the torsion $T^{\mu}$ to the scalar field produces an intricate binding of the canonical momenta, which result in serious difficulties for calculating the Hamiltonian.

It is clear that further research needs to be done in order to understand the implications of the additional d.o.f. of the theory. A simple strategy to identify it would be to resort to solutions to $f(T)$ gravity in $2+1$ dimensions, where neither TEGR nor GR possess genuine d.o.f. This issue will be addressed in future work [73].

\section{ACKNOWLEDGMENTS}

The authors are indebted to C. Bejarano and F. Fiorini for many discussions and constant support. M. J. G. would like to thank for feedback and fruitful discussions D. Blixt, M. Hohmann, T. Koivisto, M. Krššák, S. PérezBergliaffa, C. Pfeifer and E. N. Saridakis, and especially to A. Golovnev for constructive criticism. M. J. G. acknowledges the hospitality of the organizers of the Teleparallel Gravity Worskhop in Tartu 2018, where part of this work was completed, and to the participants of the workshop for helpful discussion on the results of [42]. M. J. G. was partially supported by ICTP. The work of both authors was supported by Consejo Nacional de Investigaciones Científicas y Técnicas (CONICET) and Universidad de Buenos Aires. R.F. is a member of Carrera del Investigador Científico (CONICET, Argentina). 
[1] R. Ferraro and F. Fiorini, Modified teleparallel gravity: Inflation without inflaton, Phys. Rev. D 75, 084031 (2007).

[2] R. Ferraro and F. Fiorini, On Born-Infeld gravity in Weitzenbock spacetime, Phys. Rev. D 78, 124019 (2008).

[3] F. Fiorini and R. Ferraro, A type of Born-Infeld regular gravity and its cosmological consequences, Int. J. Mod. Phys. A 24, 1686 (2009).

[4] R. Ferraro and F. Fiorini, Born-Infeld determinantal gravity and the taming of the conical singularity in 3-dimensional spacetime, Phys. Lett. B 692, 206 (2010).

[5] R. Ferraro and F. Fiorini, The regular cosmic string in BornInfeld gravity, J. Phys. Conf. Ser. 314, 012114 (2011).

[6] F. Fiorini, Nonsingular Promises from Born-Infeld Gravity, Phys. Rev. Lett. 111, 041104 (2013).

[7] F. Fiorini and N. Vattuone, An analysis of Born-Infeld determinantal gravity in Weitzenböck spacetime, Phys. Lett. B 763, 45 (2016).

[8] F. Fiorini, Primordial brusque bounce in Born-Infeld determinantal gravity, Phys. Rev. D 94, 024030 (2016).

[9] G. R. Bengochea and R. Ferraro, Dark torsion as the cosmic speed-up, Phys. Rev. D 79, 124019 (2009).

[10] P. Wu and H. W. Yu, Observational constraints on $f(T)$ theory, Phys. Lett. B 693, 415 (2010).

[11] R. Myrzakulov, Accelerating Universe from $F(T)$ gravity, Eur. Phys. J. C 71, 1752 (2011).

[12] P. Wu and H.W. Yu, The dynamical behavior of $f(T)$ theory, Phys. Lett. B 692, 176 (2010).

[13] R. J. Yang, New types of $f(T)$ gravity, Eur. Phys. J. C 71, 1797 (2011).

[14] K. Bamba, C.-Q. Geng, and C.-C. Lee, Comment on "Einstein's other gravity and the acceleration of the Universe", arXiv:1008.4036.

[15] R.-J. Yang, Conformal transformation in $f(T)$ theories, Europhys. Lett. 93, 60001 (2011).

[16] S.-H. Chen, J. B. Dent, S. Dutta, and E. N. Saridakis, Cosmological perturbations in $f(T)$ gravity, Phys. Rev. D 83, 023508 (2011).

[17] G. R. Bengochea, Observational information for $f(T)$ theories and dark torsion, Phys. Lett. B 695, 405 (2011).

[18] K. Bamba, C.-Q. Geng, C.-C. Lee, and L.-W. Luo, Equation of state for dark energy in $f(T)$ gravity, J. Cosmol. Astropart. Phys. 01 (2011) 021.

[19] B. Li, T. P. Sotiriou, and J. D. Barrow, $f(T)$ gravity and local Lorentz invariance, Phys. Rev. D 83, 064035 (2011).

[20] R. Ferraro and F. Fiorini, Non trivial frames for $f(T)$ theories of gravity and beyond, Phys. Lett. B 702, 75 (2011).

[21] T. P. Sotiriou, B. Li, and J. D. Barrow, Generalizations of teleparallel gravity and local Lorentz symmetry, Phys. Rev. D 83, 104030 (2011).

[22] B. Li, T. P. Sotiriou, and J. D. Barrow, Large-scale structure in $f(T)$ gravity, Phys. Rev. D 83, 104017 (2011).

[23] M. Li, R.-X. Miao, and Y.-G. Miao, Degrees of freedom of $f(T)$ gravity, J. High Energy Phys. 07 (2011) 108.

[24] R. Ferraro and F. Fiorini, Spherically symmetric static spacetimes in vacuum $f(T)$ gravity, Phys. Rev. D 84, 083518 (2011).

[25] R. Ferraro and F. Fiorini, Cosmological frames for theories with absolute parallelism, Int. J. Mod. Phys. Conf. Ser. 03, 227 (2011).
[26] R. Ferraro, $f(R)$ and $f(T)$ theories of modified gravity, AIP Conf. Proc. 471, 103 (2012).

[27] K. Bamba, R. Myrzakulov, S. Nojiri, and S. D. Odintsov, Reconstruction of $f(T)$ gravity: Rip cosmology, finite-time future singularities and thermodynamics, Phys. Rev. D 85, 104036 (2012).

[28] K. Izumi and Y. C. Ong, Cosmological perturbation in $f(T)$ gravity revisited, J. Cosmol. Astropart. Phys. 06 (2013) 029.

[29] F. Fiorini, P. A. González, and Y. Vásquez, Compact extra dimensions in cosmologies with $f(T)$ structure, Phys. Rev. D 89, 024028 (2014).

[30] K. Bamba, S. D. Odintsov, and D. Sáez-Gómez, Conformal symmetry and accelerating cosmology in teleparallel gravity, Phys. Rev. D 88, 084042 (2013).

[31] G. G. L. Nashed, Local Lorentz transformation and exact solution in $f(T)$ gravity theories, Astrophys. Space Sci. 348, 591 (2013).

[32] P. Chen, K. Izumi, J. M. Nester, and Y. C. Ong, Remnant symmetry, propagation and evolution in $f(T)$ gravity, Phys. Rev. D 91, 064003 (2015).

[33] C. Bejarano, R. Ferraro, and M. J. Guzmán, Kerr geometry in $f(T)$ gravity, Eur. Phys. J. C 75, 77 (2015).

[34] R. Ferraro and F. Fiorini, Remnant group of local Lorentz transformations in $f(T)$ theories, Phys. Rev. D 91, 064019 (2015).

[35] M. Wright, Conformal transformations in modified teleparallel theories of gravity revisited, Phys. Rev. D 93, 103002 (2016).

[36] G. Farrugia, J. L. Said, and M. L. Ruggiero, Solar system tests in $f(T)$ gravity, Phys. Rev. D 93, 104034 (2016).

[37] R. C. Nunes, S. Pan, and E. N. Saridakis, New observational constraints on $f(T)$ gravity from cosmic chronometers, J. Cosmol. Astropart. Phys. 08 (2016) 011.

[38] C. Bejarano, R. Ferraro, and M. J. Guzmán, McVittie solution in $f(T)$ gravity, Eur. Phys. J. C 77, 825 (2017).

[39] A. Golovnev, Introduction to teleparallel gravities, arXiv:1801.06929.

[40] M. A. Skugoreva and A. V. Toporensky, On Kasner solution in Bianchi I $f(T)$ cosmology, Eur. Phys. J. C 78, 377 (2018).

[41] M. Hohmann, L. Jarv, and U. Ualikhanova, Dynamical systems approach and generic properties of $f(T)$ cosmology, Phys. Rev. D 96, 043508 (2017).

[42] R. Ferraro and M. J. Guzmán, Hamiltonian formalism for $f(T)$ gravity, Phys. Rev. D 97, 104028 (2018).

[43] Y.C. Ong, Degrees of freedom and problems in $f(T)$ gravity, Int. J. Geom. Methods Mod. Phys. 15, 1850139 (2018).

[44] A. Golovnev and T. Koivisto, Cosmological perturbations in modified teleparallel gravity models, J. Cosmol. Astropart. Phys. 11 (2018) 012.

[45] P. A. González and Y. Vásquez, Teleparallel equivalent of Lovelock gravity, Phys. Rev. D 92, 124023 (2015).

[46] S. Bahamonde and C. G. Böhmer, Modified teleparallel theories of gravity: Gauss-Bonnet and trace extensions, Eur. Phys. J. C 76, 578 (2016).

[47] S. Bahamonde and S. Capozziello, Noether symmetry approach in $f(T, B)$ teleparallel cosmology, Eur. Phys. J. C 77, 107 (2017). 
[48] S. Bahamonde, C. G. Böhmer, and M. Krššák, New classes of modified teleparallel gravity models, Phys. Lett. B 775, 37 (2017).

[49] M. Hohmann, L. Järv, M. Krššák, and C. Pfeifer, Teleparallel theories of gravity as analogue of nonlinear electrodynamics, Phys. Rev. D 97, 104042 (2018).

[50] Y. C. Ong and J. M. Nester, Counting components in the Lagrange multiplier formulation of teleparallel theories, Eur. Phys. J. C 78, 568 (2018).

[51] M. Krššák and E. N. Saridakis, The covariant formulation of $f(T)$ gravity, Classical Quantum Gravity 33, 115009 (2016).

[52] A. Golovnev, T. Koivisto, and M. Sandstad, On the covariance of teleparallel gravity theories, Classical Quantum Gravity 34, 145013 (2017).

[53] M. Hohmann, L. Järv, and U. Ualikhanova, Covariant formulation of scalar-torsion gravity, Phys. Rev. D 97, 104011 (2018).

[54] A. D. Dolgov and M. Kawasaki, Can modified gravity explain accelerated cosmic expansion?, Phys. Lett. B 573, 1 (2003).

[55] S. Nojiri and S. D. Odintsov, Modified gravity with negative and positive powers of the curvature: Unification of the inflation and of the cosmic acceleration, Phys. Rev. D 68 , 123512 (2003).

[56] V. Faraoni, Matter instability in modified gravity, Phys. Rev. D 74, 104017 (2006).

[57] G. J. Olmo, Limit to general relativity in $f(R)$ theories of gravity, Phys. Rev. D 75, 023511 (2007).

[58] T. P. Sotiriou and V. Faraoni, $f(R)$ theories of gravity, Rev. Mod. Phys. 82, 451 (2010).

[59] J. D. Barrow and S. Cotsakis, Inflation and the conformal structure of higher order gravity theories, Phys. Lett. B 214, 515 (1988).
[60] K. I. Maeda, Towards the Einstein-Hilbert action via conformal transformation, Phys. Rev. D 39, 3159 (1989).

[61] T. Chiba, $1 / R$ gravity and scalar-tensor gravity, Phys. Lett. B 575, 1 (2003).

[62] V. Faraoni and E. Gunzig, Einstein frame or Jordan frame?, Int. J. Theor. Phys. 38, 217 (1999).

[63] A. Bhadra, K. Sarkar, D. P. Datta, and K. K. Nandi, BransDicke theory: Jordan versus Einstein frame, Mod. Phys. Lett. A 22, 367 (2007).

[64] S. Capozziello, P. Martin-Moruno, and C. Rubano, Physical non-equivalence of the Jordan and Einstein frames, Phys. Lett. B 689, 117 (2010).

[65] N. Sk and A. K. Sanyal, Why scalar-tensor equivalent theories are not physically equivalent?, Int. J. Mod. Phys. D 26, 1750162 (2017).

[66] R. Ferraro and M. J. Guzmán, Hamiltonian formulation of teleparallel gravity, Phys. Rev. D 94, 104045 (2016).

[67] Y. Itin, F. W. Hehl, and Y. N. Obukhov, Premetric equivalent of general relativity: Teleparallelism, Phys. Rev. D 95, 084020 (2017).

[68] Y. Itin, Y. N. Obukhov, J. Boos, and F. W. Hehl, Premetric teleparallel theory of gravity and its local and linear constitutive law, Eur. Phys. J. C 78, 907 (2018).

[69] J. W. Maluf and J. F. da Rocha-Neto, Hamiltonian formulation of general relativity in the teleparallel geometry, Phys. Rev. D 64, 084014 (2001).

[70] K. Sundermeyer, Symmetries in Fundamental Physics (Springer, Cham, Switzerland, 2014).

[71] M. Ostrogradsky, Mem. Ac. St. Petersbourg VI 4, 385 (1850).

[72] R. P. Woodard, Ostrogradsky's theorem on Hamiltonian instability, Scholarpedia 10, 32243 (2015).

[73] C. Bejarano, R. Ferraro, and M. J. Guzmán (to be published). 